Mathematical Modelling AND AnAlysis

Volume 18 Number 5, November 2013, 631-640

http://dx.doi.org/10.3846/13926292.2013.868840

(c) Vilnius Gediminas Technical University, 2013
Publisher: Taylor\&Francis and VGTU

http://www.tandfonline.com/TMMA

Print ISSN: 1392-6292

Online ISSN: 1648-3510

\title{
An Improved Finite Element Approximation and Superconvergence for Temperature Control Problems*
}

\section{Yuelong Tang}

\author{
Department of Mathematics and Computational Science, Hunan University \\ of Science and Engineering \\ Yongzhou 425100, Hunan, China \\ E-mail: tangyuelonga@163.com
}

Received July 22, 2012; revised October 22, 2013; published online December 1, 2013

\begin{abstract}
In this paper, we consider an improved finite element approximation for temperature control problems, where the state and the adjoint state are discretized by piecewise linear functions while the control is not discretized directly. The numerical solution of the control is obtained by a projection of the adjoint state to the set of admissible controls. We derive a priori error estimates and superconvergence of second-order. Moreover, we present some numerical examples to illustrate our theoretical results.
\end{abstract}

Keywords: optimal control, finite element method, approximation, convergence analysis.

AMS Subject Classification: 49J20; 65M60; 80M10.

\section{Introduction}

Temperature control problem plays a very important role in our everyday life. For example, a refrigerator works through a cooling system very similar to the one in an air conditioning unit. It has refrigerant that it cycles through a compressor and evaporator to take heat out of the refrigerator. Normally, the system only cycles on periodically to maintain a temperature range. The system is governed by the following equation:

$$
\left\{\begin{array}{l}
-\operatorname{div}(A \nabla y)+\phi(y)=f, \quad x \in \Omega, \\
\left.y\right|_{\partial \Omega}=0
\end{array}\right.
$$

where $\Omega$ is a convex domain with a Lipschitz boundary $\partial \Omega, y$ is the temperature distribution which is maintained equal to zero along the boundary, $\phi(y)$ denotes the thermal radiation or positive temperature feedback due to chemical reactions (see e.g., [16]) and $f$ is a source term.

\footnotetext{
* This work is supported by the Foundation of Hunan Educational Committee (13C338).
} 
Then we may control the temperature distribution $y$ to come close to a given target $y_{d}$ by acting with an additional distributed source term $u$. The corresponding temperature control problem is formulated as follows:

$$
\left\{\begin{array}{l}
\min _{u \in K}\left\{\frac{1}{2}\left\|y-y_{d}\right\|^{2}+\frac{1}{2}\|u\|^{2}\right\}, \\
-\operatorname{div}(A \nabla y)+\phi(y)=f+B u, \quad x \in \Omega, \\
\left.y\right|_{\partial \Omega}=0,
\end{array}\right.
$$

where $K$ is a closed convex subset in $L^{2}(\Omega)$, the coefficient matrix $A=$ $\left(a_{i j}\right)_{n \times n} \in\left(W^{1, \infty}(\bar{\Omega})\right)^{n \times n}$, such that $(A \xi) \cdot \xi \geq c|\xi|^{2}, \forall \xi \in \mathbb{R}^{n}, f \in L^{2}(\Omega)$, and $B$ is a linear continuous operator. Moreover, $K$ is defined by

$$
K=\left\{v(x) \in L^{2}(\Omega): a \leq v(x) \leq b, \text { a.e. } x \in \Omega\right\},
$$

where $a$ and $b$ are constants.

The literature on finite element solving optimal control problems is huge. For a linear elliptic optimal control problem, a priori error estimates were investigated in [10], a posteriori error estimates based on recovery techniques have been obtained in $[9,17]$, a posteriori error estimates of residual type have been derived in [12], a superconvergence result can be seen in [14], a variational discretization method was introduced in [13], and Chen et al. considered its Legendre Galerkin spectral approximation in [4].

For semilinear elliptic optimal control problems, a priori error estimates were investigated in [1], a posteriori error estimates have been obtained in [11], a superconvergence can be seen in [3], Borzì considered a second-order discretization and multigrid solution in [2]. Recently, some adaptive algorithm and superconvergence results can be found in $[6,7,15]$. Notice that all the control variables are first-order convergence and the superconvergence of the state and the adjoint state variables are 1.5 order in the above works.

To the best of our knowledge, our work is the first to consider a numerical approximation for the semilinear elliptic temperature control problem in which both the a priori error estimates and the superconvergence are of the secondorder.

The paper is organized as follows: In Section 2, we introduce an improved finite element approximation for the model problem. In Section 3, we derive a priori error estimates. In Section 4, we obtain the superconvergence property. We present some numerical examples to confirm our theoretical results in the last section.

\section{An Improved Finite Element for the Model Problem}

For ease of exposition, we set $W=H_{0}^{1}(\Omega), U=L^{2}(\Omega),\|\cdot\|=\|\cdot\|_{L^{2}(\Omega)}$, $\|\cdot\|_{m}=\|\cdot\|_{H^{m}(\Omega)}$ and

$$
\begin{aligned}
a(y, w) & =\int_{\Omega}(A \nabla y) \cdot \nabla w, \quad \forall y, w \in W, \\
(u, w) & =\int_{\Omega} u \cdot w, \quad \forall u, w \in U .
\end{aligned}
$$


It follows from the assumptions on the coefficient matrix $A$ that

$$
a(y, y) \geq c\|y\|_{1}^{2}, \quad|a(y, w)| \leq C\|y\|_{1}\|w\|_{1}, \quad \forall y, w \in W .
$$

Then the standard weak formula for the state equation reads as follows:

$$
a(y, w)+(\phi(y), w)=(f+B u, w), \quad \forall w \in W
$$

where we assume that the function $\phi(\cdot) \in W^{2, \infty}(-R, R)$ for any $R>0$ and $\phi^{\prime}(\cdot) \geq 0$.

Then the model problem (1.1) can be restated as: Find $(y, u) \in W \times K$, such that

$$
\left\{\begin{array}{l}
\min _{u \in K}\left\{\frac{1}{2}\left\|y-y_{d}\right\|^{2}+\frac{1}{2}\|u\|^{2}\right\}, \\
a(y, w)+(\phi(y), w)=(f+B u, w), \quad \forall w \in W .
\end{array}\right.
$$

It is well known (see e.g., [10]) that the control problem (2.3) has a solution $(y, u) \in W \times K$, and that if the pair $(y, u) \in W \times K$ is the solution of (2.3), then there is an adjoint state $p \in W$ such that the triplet $(y, p, u) \in W \times W \times K$ satisfies the following optimal conditions:

$$
\begin{aligned}
a(y, w)+(\phi(y), w) & =(f+B u, w), \quad \forall w \in W, \\
a(q, p)+\left(\phi^{\prime}(y) p, q\right) & =\left(y_{d}-y, q\right), \quad \forall q \in W, \\
\left(u-B^{*} p, v-u\right) & \geq 0, \quad \forall v \in K,
\end{aligned}
$$

where $B^{*}$ is the adjoint operator of $B$.

We introduce the following pointwise projection operator:

$$
\Pi_{[a, b]}(g(x))=\max (a, \min (b, g(x))) .
$$

As in $[13,14]$, it is easy to prove the following lemma:

Lemma 1. Let $(y, p, u)$ be the solution of (2.4)-(2.6). Then we have

$$
u=\Pi_{[a, b]}\left(B^{*} p\right) .
$$

Let $\mathcal{T}^{h}$ be a regular triangulation of $\Omega$, such that $\bar{\Omega}=\cup_{\tau \in \mathcal{T}^{h}} \bar{\tau}$. Let $h=$ $\max _{\tau \in \mathcal{T} h}\left\{h_{\tau}\right\}$, where $h_{\tau}$ denotes the diameter of the element $\tau$. Associated with $\mathcal{T}^{h}$ is a finite dimensional subspace $S^{h}$ of $C(\bar{\Omega})$, such that $\left.\chi\right|_{\tau}$ are polynomials of m-order $(m \leq 1)$ for all $\chi \in S^{h}$ and $\tau \in \mathcal{T}^{h}$. Let $W^{h}=\left\{v_{h} \in S^{h}:\left.v_{h}\right|_{\partial \Omega}=0\right\}$. It is easy to see that $W^{h} \subset W$.

Then an improved finite element approximation scheme of (2.3) is as follows: Find $\left(y_{h}, u_{h}\right) \in W^{h} \times K$, such that

$$
\left\{\begin{array}{l}
\min _{u_{h} \in K}\left\{\frac{1}{2}\left\|y_{h}-y_{d}\right\|^{2}+\frac{1}{2}\left\|u_{h}\right\|^{2}\right\}, \\
a\left(y_{h}, w_{h}\right)+\left(\phi\left(y_{h}\right), w_{h}\right)=\left(f+B u_{h}, w_{h}\right), \quad \forall w_{h} \in W^{h} .
\end{array}\right.
$$


It follows (see e.g., [11]) that the control problem (2.9) has a solution $\left(y_{h}, u_{h}\right) \in$ $W^{h} \times K$, and that if the pair $\left(y_{h}, u_{h}\right) \in W^{h} \times K$ is the solution of (2.9), then there is an adjoint state $p_{h} \in W^{h}$ such that the triplet $\left(y_{h}, p_{h}, u_{h}\right) \in$ $W^{h} \times W^{h} \times K$ satisfies the following optimal conditions:

$$
\begin{aligned}
a\left(y_{h}, w_{h}\right)+\left(\phi\left(y_{h}\right), w_{h}\right) & =\left(f+B u_{h}, w_{h}\right), \quad \forall w_{h} \in W^{h}, \\
a\left(q_{h}, p_{h}\right)+\left(\phi^{\prime}\left(y_{h}\right) p_{h}, q_{h}\right) & =\left(y_{d}-y_{h}, q_{h}\right), \quad \forall q_{h} \in W^{h}, \\
\left(u_{h}-B^{*} p_{h}, v-u_{h}\right) & \geq 0, \quad \forall v \in K .
\end{aligned}
$$

Similar to Lemma 1 , as in $[13,14]$, it is easy to show the following lemma:

Lemma 2. Let $\left(y_{h}, p_{h}, u_{h}\right)$ be the solution of (2.10)-(2.12). Then we have

$$
u_{h}=\Pi_{[a, b]}\left(B^{*} p_{h}\right) .
$$

\section{A Priori Error Estimates}

We introduce some intermediate variables. Let $\left(y\left(u_{h}\right), p\left(u_{h}\right)\right)$ satisfy the following system:

$$
\begin{aligned}
a\left(y\left(u_{h}\right), w\right)+\left(\phi\left(y\left(u_{h}\right)\right), w\right) & =\left(f+B u_{h}, w\right), \quad \forall w \in W, \\
a\left(q, p\left(u_{h}\right)\right)+\left(\phi^{\prime}\left(y\left(u_{h}\right)\right) p\left(u_{h}\right), q\right) & =\left(y_{d}-y\left(u_{h}\right), q\right), \quad \forall q \in W .
\end{aligned}
$$

Just for ease of exposition, let

$$
J(u)=\frac{1}{2}\left\|y-y_{d}\right\|^{2}+\frac{1}{2}\|u\|^{2},
$$

and $J^{\prime}(u)$ is the Fréchet derivative of $J(u)$ at $u$. It is easy to prove that

$$
\begin{aligned}
\left(J^{\prime}(u), v\right) & =\left(u-B^{*} p, v\right), \quad \forall v \in K, \\
\left(J^{\prime}\left(u_{h}\right), v\right) & =\left(u_{h}-B^{*} p\left(u_{h}\right), v\right), \quad \forall v \in K .
\end{aligned}
$$

Lemma 3. [5] Let $\pi_{h}$ be the standard Lagrange interpolation operator. For $m=0$ or $1, q>\frac{n}{2}$ and $\forall v \in W^{2, q}(\Omega)$, we have

$$
\left|v-\pi_{h} v\right|_{W^{m, q}(\Omega)} \leq C h^{2-m}|v|_{W^{2, q}(\Omega)} .
$$

Lemma 4. Let $\left(y_{h}, p_{h}, u_{h}\right)$ and $\left(y\left(u_{h}\right), p\left(u_{h}\right)\right)$ be the solutions of $(2.10)-(2.12)$ and (3.1)-(3.2), respectively. Assume that $p\left(u_{h}\right), y\left(u_{h}\right) \in H^{2}(\Omega)$. Then there exists a constant $C$ independent of $h$ such that

$$
\left\|y\left(u_{h}\right)-y_{h}\right\|_{1}+\left\|p\left(u_{h}\right)-p_{h}\right\|_{1} \leq C h .
$$

Proof. According to (2.1), (2.10), (3.1), $\phi^{\prime}(\cdot) \geq 0$ and Cauchy's inequality with $\varepsilon$, we have

$$
\begin{aligned}
c \| & y\left(u_{h}\right)-y_{h} \|_{1}^{2} \\
& \leq a\left(y\left(u_{h}\right)-y_{h}, y\left(u_{h}\right)-y_{h}\right)+\left(\phi\left(y\left(u_{h}\right)\right)-\phi\left(y_{h}\right), y\left(u_{h}\right)-y_{h}\right) \\
& =a\left(y\left(u_{h}\right)-\pi_{h} y\left(u_{h}\right), y\left(u_{h}\right)-y_{h}\right)+\left(\phi\left(y\left(u_{h}\right)\right)-\phi\left(y_{h}\right), y\left(u_{h}\right)-\pi_{h} y\left(u_{h}\right)\right) \\
& \leq C\left\|y\left(u_{h}\right)-y_{h}\right\|_{1}\left\|y\left(u_{h}\right)-\pi_{h} y\left(u_{h}\right)\right\|_{1}+C\left\|y\left(u_{h}\right)-y_{h}\right\|\left\|\pi_{h} y\left(u_{h}\right)-y_{h}\right\| \\
& \leq 2 \varepsilon\left\|y\left(u_{h}\right)-y_{h}\right\|_{1}^{2}+\frac{C}{2 \varepsilon}\left\|y\left(u_{h}\right)-\pi_{h} y\left(u_{h}\right)\right\|_{1}^{2} .
\end{aligned}
$$


Note that $y\left(u_{h}\right) \in H^{2}(\Omega)$, by using Lemma 3, we obtain

$$
\left\|y\left(u_{h}\right)-\pi_{h} y\left(u_{h}\right)\right\|_{1} \leq C h\left\|y\left(u_{h}\right)\right\|_{2} .
$$

Let $2 \varepsilon<c$, it follows from (3.4)-(3.5) that

$$
\left\|y\left(u_{h}\right)-y_{h}\right\|_{1} \leq C h .
$$

Similarly, we can prove that

$$
\left\|p\left(u_{h}\right)-p_{h}\right\|_{1} \leq C h .
$$

Then (3.3) follows from (3.6)-(3.7).

We introduce the following auxiliary problems:

$$
\begin{aligned}
& -\operatorname{div}\left(A^{*} \nabla \xi\right)+\Phi \xi=F_{1}, \quad \text { in } \Omega,\left.\quad \xi\right|_{\partial \Omega}=0, \\
& -\operatorname{div}(A \nabla \zeta)+\phi^{\prime}\left(y\left(u_{h}\right)\right) \zeta=F_{2}, \quad \text { in } \Omega,\left.\quad \zeta\right|_{\partial \Omega}=0 \text {, }
\end{aligned}
$$

where

$$
\Phi= \begin{cases}\frac{\phi\left(y\left(u_{h}\right)\right)-\phi\left(y_{h}\right)}{y\left(u_{h}\right)-y_{h}}, & y\left(u_{h}\right) \neq y_{h}, \\ \phi^{\prime}\left(y_{h}\right), & y\left(u_{h}\right)=y_{h} .\end{cases}
$$

From the regularity estimates (see e.g., [16]), we obtain

$$
\|\xi\|_{2} \leq C\left\|F_{1}\right\|, \quad\|\zeta\|_{2} \leq C\left\|F_{2}\right\| .
$$

By using the Aubin-Nitsche technique, it is easy to prove the following lemma:

Lemma 5. Let $\left(y_{h}, p_{h}, u_{h}\right)$ and $\left(y\left(u_{h}\right), p\left(u_{h}\right)\right)$ be the solutions of (2.10)-(2.12) and (3.1)-(3.2), respectively. Assume that $p\left(u_{h}\right), y\left(u_{h}\right) \in H^{2}(\Omega)$. Then there exists a constant $C$ independent of $h$ such that

$$
\left\|y\left(u_{h}\right)-y_{h}\right\|+\left\|p\left(u_{h}\right)-p_{h}\right\| \leq C h^{2} .
$$

Lemma 6. Let $(y, p, u)$ and $\left(y_{h}, p_{h}, u_{h}\right)$ be the solutions of $(2.4)-(2.6)$ and (2.10)-(2.12), respectively. Assume that all the conditions in Lemma 5 are valid. Then there exists a constant $C$ independent of $h$ such that

$$
\left\|u-u_{h}\right\| \leq C h^{2}
$$

Proof. It is clear that

$$
\left(J^{\prime}(v)-J^{\prime}(u), v-u\right) \geq c\|v-u\|^{2}, \quad \forall v, u \in K .
$$

By using (2.6) and (2.12), we have

$$
\begin{aligned}
c\left\|u-u_{h}\right\|^{2} & \leq\left(J^{\prime}(u)-J^{\prime}\left(u_{h}\right), u-u_{h}\right) \\
& =\left(u-B^{*} p, u-u_{h}\right)-\left(u_{h}-B^{*} p\left(u_{h}\right), u-u_{h}\right) \\
& \leq\left(B^{*} p\left(u_{h}\right)-B^{*} p_{h}, u-u_{h}\right) \\
& \leq C\left\|p\left(u_{h}\right)-p_{h}\right\|\left\|u-u_{h}\right\| .
\end{aligned}
$$

From (3.10) and (3.13), we derive (3.11). 
By combining Lemmas 3-6, we can prove the following main result.

Theorem 1. Let $(y, p, u)$ and $\left(y_{h}, p_{h}, u_{h}\right)$ be the solutions of (2.4)-(2.6) and (2.10)-(2.12), respectively. Assume that all the conditions in Lemmas 4-6 are valid. Then, we have

$$
\left\|u-u_{h}\right\|+\left\|y-y_{h}\right\|+\left\|p-p_{h}\right\| \leq C h^{2} .
$$

\section{Superconvergence Property}

We introduce an elliptic projection operator $P_{h}: W \rightarrow W^{h}$, which satisfies: for any $\phi \in W$

$$
a\left(\phi-P_{h} \phi, w_{h}\right)=0, \quad \forall w_{h} \in W^{h} .
$$

It has the following approximation property (see e.g., [18]):

$$
\left\|\phi-P_{h} \phi\right\| \leq C h^{2}\|\phi\|_{2}, \quad \forall \phi \in H^{2}(\Omega) .
$$

Then we consider the superconvergence property between elliptic projections and numerical solutions of the state and the adjoint state.

Theorem 2. Let $(y, p, u)$ and $\left(y_{h}, p_{h}, u_{h}\right)$ be the solutions of $(2.4)-(2.6)$ and (2.10)-(2.12), respectively. Then there exists a constant $C$ independent of $h$ such that

$$
\left\|P_{h} y-y_{h}\right\|_{1}+\left\|P_{h} p-p_{h}\right\|_{1} \leq C h^{2} .
$$

Proof. From (2.4) and (2.10), we get

$$
a\left(y-y_{h}, w_{h}\right)+\left(\phi(y)-\phi\left(y_{h}\right), w_{h}\right)=\left(B\left(u-u_{h}\right), w_{h}\right), \quad \forall w_{h} \in W^{h} .
$$

According to the definition of $P_{h}$, we have

$$
a\left(P_{h} y-y_{h}, w_{h}\right)+\left(\phi(y)-\phi\left(y_{h}\right), w_{h}\right)=\left(B\left(u-u_{h}\right), w_{h}\right), \quad \forall w_{h} \in W^{h} .
$$

By selecting $w_{h}=P_{h} y-y_{h}$ and using (2.1) and the assumed conditions on $\phi(\cdot)$, we obtain

$$
\begin{aligned}
c\left\|P_{h} y-y_{h}\right\|_{1}^{2} & \leq a\left(P_{h} y-y_{h}, P_{h} y-y_{h}\right)+\left(\phi\left(P_{h} y\right)-\phi\left(y_{h}\right), P_{h} y-y_{h}\right) \\
& =\left(\phi\left(P_{h} y\right)-\phi(y), P_{h} y-y_{h}\right)+\left(B\left(u-u_{h}\right), P_{h} y-y_{h}\right) \\
& \leq C\left(\left\|P_{h} y-y\right\|+\left\|u-u_{h}\right\|\right)\left\|P_{h} y-y_{h}\right\| \\
& \leq C h^{2}\left\|P_{h} y-y_{h}\right\| .
\end{aligned}
$$

Hence,

$$
\left\|P_{h} y-y_{h}\right\|_{1} \leq C h^{2} .
$$

Similarly, we can prove that

$$
\left\|P_{h} p-p_{h}\right\|_{1} \leq C h^{2} .
$$

Then (4.3) follows from (4.4)-(4.5). 
Table 1. Numerical results, Example 1.

\begin{tabular}{lccccc}
\hline Mesh & $\left\|u-u_{h}\right\|$ & $\left\|y-y_{h}\right\|$ & $\left\|p-p_{h}\right\|$ & $\left\|P_{h} y-y_{h}\right\|_{1}$ & $\left\|P_{h} p-p_{h}\right\|_{1}$ \\
\hline $10 \times 10$ & $3.10200 \mathrm{e}-02$ & $5.27023 \mathrm{e}-02$ & $5.03458 \mathrm{e}-02$ & $5.43093 \mathrm{e}-02$ & $6.16469 \mathrm{e}-02$ \\
$20 \times 20$ & $7.78194 \mathrm{e}-03$ & $1.38042 \mathrm{e}-02$ & $1.31083 \mathrm{e}-02$ & $1.41742 \mathrm{e}-02$ & $1.62494 \mathrm{e}-02$ \\
$40 \times 40$ & $2.00103 \mathrm{e}-03$ & $3.49306 \mathrm{e}-03$ & $3.31053 \mathrm{e}-03$ & $3.58245 \mathrm{e}-03$ & $4.12064 \mathrm{e}-03$ \\
$80 \times 80$ & $5.01440 \mathrm{e}-04$ & $8.75888 \mathrm{e}-04$ & $8.29739 \mathrm{e}-04$ & $8.98110 \mathrm{e}-04$ & $1.03390 \mathrm{e}-03$ \\
$160 \times 160$ & $1.25846 \mathrm{e}-04$ & $2.19143 \mathrm{e}-04$ & $2.07566 \mathrm{e}-04$ & $2.24679 \mathrm{e}-04$ & $2.58711 \mathrm{e}-04$ \\
\hline
\end{tabular}

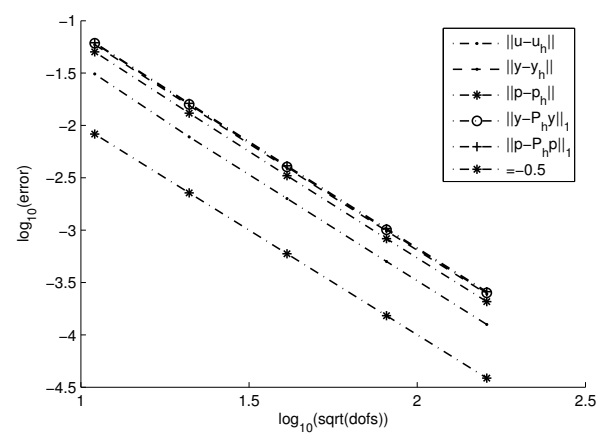

Figure 1. The order of convergence, Example 1.

\section{$5 \quad$ Numerical Experiment}

In this section, optimal control problems were solved numerically with codes developed by using AFEPack. This package is freely available and the details can be found in [8]. The discretization has been described in Section 2 .

Let $\Omega=[0,1] \times[0,1]$ and $B=I$, we solve the following semilinear optimal control problem:

$$
\left\{\begin{array}{l}
\min _{u \in K}\left\{\frac{1}{2}\left\|y-y_{d}\right\|^{2}+\frac{1}{2}\|u\|^{2}\right\} \\
-\operatorname{div}(A \nabla y)+\phi(y)=f+B u, \quad x \in \Omega \\
\left.y\right|_{\partial \Omega}=0
\end{array}\right.
$$

Example 1. The data are as follows:

$$
\begin{aligned}
& A=I, \quad \phi(y)=y^{3}, \quad a=-0.5, \quad b=0.5, \\
& p(x)=\sin \left(2 \pi x_{1}\right) \sin \left(2 \pi x_{2}\right), \quad y(x)=p(x), \\
& u(x)=\min (0.5, \max (-0.5, p(x))), \\
& f(x)=-\operatorname{div}(A \nabla y(x))+\phi(y(x))-u(x), \\
& y_{d}(x)=y(x)-\operatorname{div}\left(A^{*} \nabla p(x)\right)+\phi^{\prime}(y(x)) p(x) .
\end{aligned}
$$

Some numerical results based on a sequence of uniformly refined meshes are shown in Table 1. 

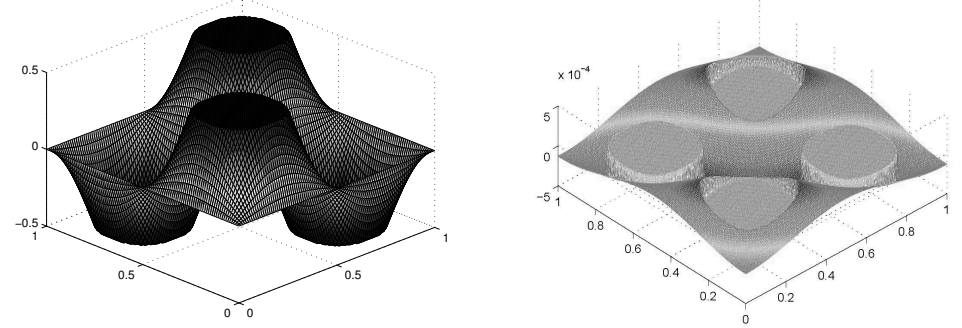

Figure 2. The exact solution $u$ (left) and the error $u-u_{h}$ (right), Example 1 .

Table 2. Numerical results, Example 2.

\begin{tabular}{lccccc}
\hline Mesh & $\left\|u-u_{h}\right\|$ & $\left\|y-y_{h}\right\|$ & $\left\|p-p_{h}\right\|$ & $\left\|P_{h} y-y_{h}\right\|_{1}$ & $\left\|P_{h} p-p_{h}\right\|_{1}$ \\
\hline $10 \times 10$ & $2.65120 \mathrm{e}-02$ & $5.30353 \mathrm{e}-02$ & $5.38607 \mathrm{e}-02$ & $5.59887 \mathrm{e}-02$ & $5.59896 \mathrm{e}-02$ \\
$20 \times 20$ & $6.23877 \mathrm{e}-03$ & $1.39308 \mathrm{e}-02$ & $1.41608 \mathrm{e}-02$ & $1.40492 \mathrm{e}-02$ & $1.41264 \mathrm{e}-02$ \\
$40 \times 40$ & $1.56441 \mathrm{e}-03$ & $3.52693 \mathrm{e}-03$ & $3.52612 \mathrm{e}-03$ & $3.56435 \mathrm{e}-03$ & $3.58241 \mathrm{e}-03$ \\
$80 \times 80$ & $3.92635 \mathrm{e}-04$ & $8.64530 \mathrm{e}-04$ & $8.69441 \mathrm{e}-04$ & $8.76090 \mathrm{e}-04$ & $8.80613 \mathrm{e}-04$ \\
$160 \times 160$ & $9.82587 \mathrm{e}-05$ & $2.11316 \mathrm{e}-04$ & $2.15043 \mathrm{e}-04$ & $2.19025 \mathrm{e}-04$ & $2.20152 \mathrm{e}-04$ \\
\hline
\end{tabular}

We show the relationship between $\log _{10}($ error $)$ and $\log _{10}(\operatorname{sqrt}($ dofs $))$ in Figure 1. It is clear that $\left\|u-u_{h}\right\|=O\left(h^{2}\right),\left\|y-y_{h}\right\|=O\left(h^{2}\right),\left\|p-p_{h}\right\|=O\left(h^{2}\right)$, $\left\|y-P_{h} y\right\|_{1}=O\left(h^{2}\right),\left\|p-P_{h} p\right\|_{1}=O\left(h^{2}\right)$. In Figure 2, we plot the exact solution $u$ and the error $u-u_{h}$.

Example 2. The data are as follows:

$$
\begin{aligned}
& A=I, \quad \phi(y)=e^{y}, \quad a=-1, \quad b=0.5, \\
& p(x)=\left(1-4 x_{1} x_{2}\right) \sin \left(2 \pi x_{1}\right) \sin \left(2 \pi x_{2}\right), \quad y(x)=p(x), \\
& u(x)=\min (0.5, \max (-1, p(x))), \\
& f(x)=-\operatorname{div}(A \nabla y(x))+\phi(y(x))-u(x), \\
& y_{d}(x)=y(x)-\operatorname{div}\left(A^{*} \nabla p(x)\right)+\phi^{\prime}(y(x)) p(x) .
\end{aligned}
$$

In Table 2, we have shown some numerical results on a sequential meshes. The second - order convergence of errors $\left\|u-u_{h}\right\|,\left\|y-y_{h}\right\|,\left\|p-p_{h}\right\|,\left\|y-P_{h} y\right\|_{1}$, and $\left\|p-P_{h} p\right\|_{1}$ can be seen in Figure 3. The exact solution $u$ and the error $u-u_{h}$ are shown in Figure 4 .

The above numerical examples confirm $\left\|u-u_{h}\right\|=O\left(h^{2}\right),\left\|y-P_{h} y\right\|_{1}=$ $O\left(h^{2}\right),\left\|p-P_{h} p\right\|_{1}=O\left(h^{2}\right)$. But in [3], the authors have just derived the estimates $\left\|u_{h}-G_{h} u_{h}\right\|=O\left(h^{\frac{3}{2}}\right),\left\|y-P_{h} y\right\|_{1}=O\left(h^{\frac{3}{2}}\right),\left\|p-P_{h} p\right\|_{1}=O\left(h^{\frac{3}{2}}\right)$. Hence, our method is much more efficient.

\section{Conclusions}

We have investigated an improved finite element approximation for semilinear temperature control problem and have obtained a priori error estimates and 


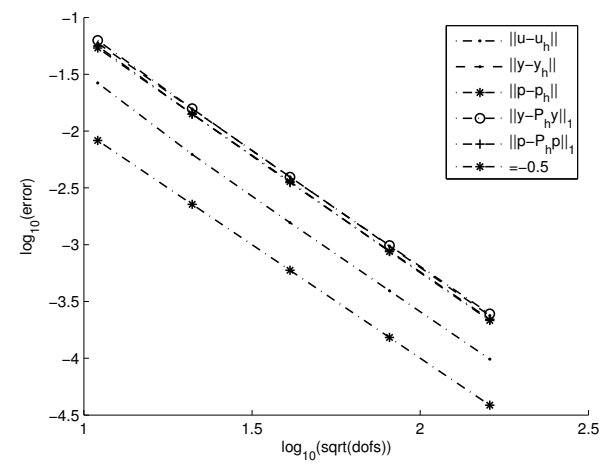

Figure 3. The order of convergence, Example 2.
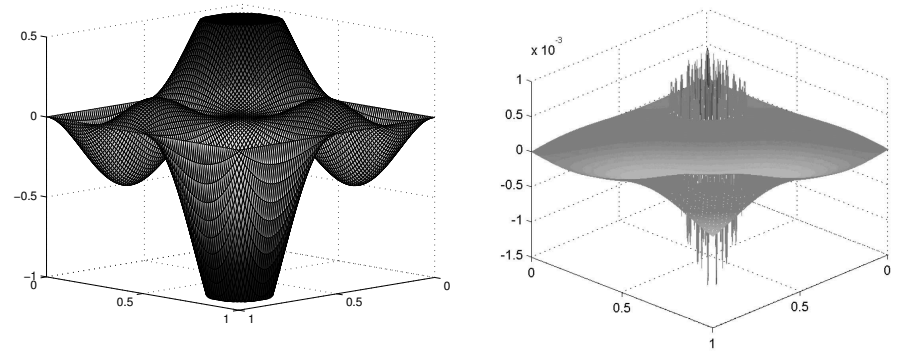

Figure 4. The exact solution $u$ (left) and the error $u-u_{h}$ (right), Example 2 .

superconvergence of the second-order. Our analysis for the semilinear temperature control problem seems to be new, and these results can be extended to general convex problems.

\section{References}

[1] N. Arada, E. Casas and F. Troltzsch. Error estimates for the numerical approximation of a semilinear elliptic control problem. Comput. Optim. Appl., 23:201-229, 2002. http://dx.doi.org/10.1023/A:1020576801966.

[2] A. Borzì. High-order discretization and multigrid solution of elliptic nonlinear constrained control problems. J. Comput. Appl. Math., 200:67-85, 2005. http://dx.doi.org/10.1016/j.cam.2005.12.023.

[3] Y. Chen and Y. Dai. Superconvergence for optimal control problems gonverned by semi-linear elliptic equations. J. Sci. Comput., 39:206-221, 2009. http://dx.doi.org/10.1007/s10915-008-9258-9.

[4] Y. Chen, N. Yi and W. Liu. A Legendre Galerkin spectral method for optimal control problems governed by elliptic equations. SIAM J. Numer. Anal., 46(5):2254-2275, 2008. http://dx.doi.org/10.1137/070679703.

[5] P. Ciarlet. The Finite Element Method for Elliptic Problems. North-Holland, 1978. 
[6] R. Hoppe and M. Kieweg. Adaptive finite element methods for mixed controlstate constrained optimal control problems for elliptic boundary value problems. Comput. Optim. Appl., 46:511-533, 2010. http://dx.doi.org/10.1007/s10589-008-9195-4.

[7] C. Hou, Y. Chen and Z. Lu. Superconvergence property of finite element methods for parabolic optimal control problems. J. Ind. Manag. Optim., 7(4):927-945, 2011. http://dx.doi.org/10.3934/jimo.2011.7.927.

[8] R. Li. The AFEPack Handbook. http://circus.math.pku.edu.cn/afepack/, 2006.

[9] R. Li, W. Liu and N. Yan. A posteriori error estimates of recovery type for distributed convex optimal control problems. J. Sci. Comput., 33:155-182, 2007. http://dx.doi.org/10.1007/s10915-007-9147-7.

[10] J. Lions and E. Magenes. Non Homogeneous Boundary Value Problems and Applications. Springer-Verlag, 1972.

[11] W. Liu and N. Yan. A posteriori error estimates for control problems governed by nonlinear elliptic equations. Appl. Numer. Math., 47:173-187, 2003. http://dx.doi.org/10.1016/S0168-9274(03)00054-0.

[12] W. Liu and N. Yan. Adaptive Finite Element Methods for Optimal Control Governed by PDEs. Science Press, 2008.

[13] N. Yan M. Hinze and Z. Zhou. Variational discretization for optimal control governed by convection dominated diffusion equations. J. Comput. Math., 27:237$253,2009$.

[14] C. Meyer and A. Rosch. Superconvergence properties of optimal control problems. SIAM J. Control Optim., 43(3):970-985, 2004. http://dx.doi.org/10.1137/S0363012903431608.

[15] T. Zhang P. Huang and X. Ma. Superconvergence by L2-projection for a stabilized finite volume method for the stationary Navier-Stokes equations. Comput. Math. Appl., 62(11):4249-4257, 2011.

http://dx.doi.org/10.1016/j.camwa.2011.10.012.

[16] C. Pao. Nonlinear Parabolic and Elliptic Equations. Plenum Press, 1992.

[17] N. Yan. A posteriori error estimates of gradient recovery type for FEM of optimal control problem. Adv. Comput. Math., 19:323-336, 2003. http://dx.doi.org/10.1023/A:1022800401298.

[18] O.C. Zienkiwicz and J.Z. Zhu. The superconvergence patch recovery and a posteriori error estimates. Int. J. Numer. Meth. Eng., 33:1331-1382, 1992. http://dx.doi.org/10.1002/nme.1620330702. 